\title{
Model of structure and mechanical properties of dry granular snow
}

\author{
Vladimir N. Golubev, ${ }^{1}$ Anatoly D. Frolov ${ }^{2}$ \\ ${ }^{1}$ Department of Geography, Moscow State University, Moscow 119899, Russia \\ ${ }^{2}$ Scientific Council on Earth Cryology, Russian Academy of Sciences, 11 Fersman Street, Moscow 117312, Russia
}

\begin{abstract}
The paper discusses the verification of a previously developed structural dry-snow model based on the regular packing of isometric grains connected by rigid bonds. Using this model, we consider the probable snow-compaction mechanisms at different snow-density ranges (from new to dense snow), limited by critical densities corresponding to the real values of the structural indices of the model: texture looseness, bond rigidity and coordination number. We also obtain the analytic expression for longitudinal wave velocity and for bulk compressibility of snow as functions of density and structural indices. The calculation enables us for the first time to show the whole range of possible values of these important characteristics and to deduce the most probable configuration of the ice matrix in snow of various densities corresponding to the experimental data available on the snow-texture peculiarities and elastic-wave velocities. The model can be used to comprehend the changes in snow mechanical properties during compaction, as well as the steps by which it proceeds.
\end{abstract}

\section{INTRODUCTION}

Snow compaction and changes of physical properties result from irreversible structural changes in the open system. It is unlikely that this process develops at a constant rate throughout the density range, from soft new snow to solid ice. Evolution by stages is more typical of a complex system; its progress is determined by the fact that each successive state is related to the transformation of matrix elements at earlier stages. When compaction has progressed to a certain level, the evolution switches to another mechanism. For snow the critical density values are $\sim 140,330,550,710$ and $830 \mathrm{~kg} \mathrm{~m}^{-3}$. The compaction process is divided into a series of stages (Maeno, 1978; Patterson, 1994; Frolov and Fedyukin, 1996). Some of them were identified on the basis of changes in the compaction rate observed during constantstress controlled experiments. Many physical properties of snow, if plotted against snow density, also show noticeable changes near the "critical" points. This is true, in particular, of elastic-wave velocity, heat conductivity, electrical resistivity and strength (Frolov, 1998).

The ice matrix of snow is formed by interconnected ice grains. Any changes in grain number and in connecting elements (bonds) result in changes of snow density and of physical characteristics. It must be taken into account that different arrangements of ice grains (ice-matrix configurations) at the same density can give different physical properties. To obtain reliable data on the physical properties and texture of snow, the representative volume must include $>1000$ interconnected grains. Investigations of such large systems, aimed at finding regularities in changes of the snow texture and physical properties during compaction, would necessarily be very complicated. The use of a model which could adequately describe changes in snow physical properties on the basis of possible changes of ice-matrix configuration and of snow-texture parameters could therefore be advantageous.

This paper presents an analytical estimation of certain mechanical properties of dry granular snow and discusses the mechanisms of snow compaction obtained using a theoretical model. The model is based on a spatial regular lattice, formed by points (grains, spherical or polyhedral) connected by rigid bonds. The regular lattice is only an approximation of the actual grain arrangement which is characterized by the probability of local variations in grain dimensions and packing as well as in the thickness and length of intergranular bonds. Nevertheless, the assumption of regular packing allows us to relate the snow compaction
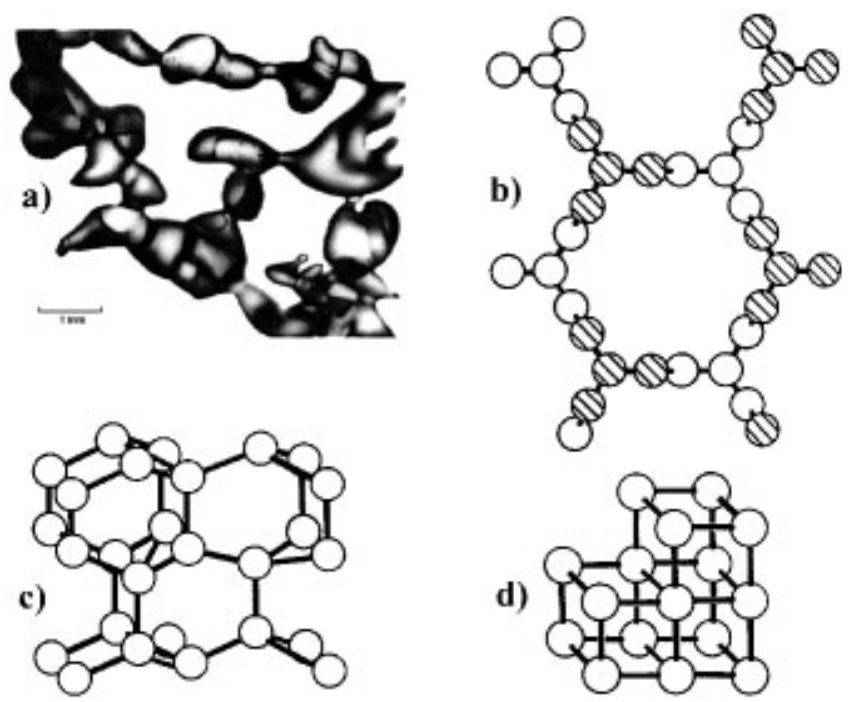

Fig. 1. Structure of natural, rounded snow ( a) and the spatial regular packing with $j=3(b), j=4(c)$ and $j=6(d)$. 
and changes in mechanical properties to the changes in textural parameters. In particular, it appears possible to relate the critical densities to successive changes in the coordination number (Golubev and Frolov, 1998).

\section{BACKGROUND}

The ice matrix of snow may be represented as a spatial regular lattice, built of spheres with diameter $D$, placed a distance $L$ apart and interconnected by rigid cylindrical bonds with diameter $d$. Figure la shows contacting ice grains forming chains in snow texture. Figure $1 \mathrm{~b}-\mathrm{d}$ give examples of spatial regular packing with coordination number $(j)$ equal to 3, 4 and 6, respectively. For these cases the snow density may be described by the following equation (Golubev, 1982; Golubev and Frolov, 1998):

$$
\rho_{\mathrm{s}}=\rho_{\mathrm{i}} A \frac{\pi}{6} \frac{1}{k^{3}}\left\{1+\frac{j}{2}\left[1.5 b^{2} k+\left(1-b^{2}\right)^{3 / 2}-1\right]\right\},
$$

where $\rho_{\mathrm{i}}$ is the density of solid ice, $k=L / D$ is the index of texture looseness, $b=d / D$ is the index of bond rigidity and $A=f(j)$ is the grain-packing factor, equal to 0.285 at $j=3$, to 0.6 at $j=4$ and to 1.0 at $j=6$.

Figure 2 shows relative snow density $\left(\rho_{\mathrm{s}} / \rho_{\mathrm{i}}\right)$ plotted against textural indexes $k$ and $b$ at fixed coordination-number values. The factors that produce the greatest variation in snow density are the coordination number $j$ and the index of texture looseness $k$. The values of $j$ and $k$ directly influence the number of grains that are principal constituents of the snow texture. Length and, consequently, mass of intergranular bonds also change with changes of $k$. The dependence of $\rho_{\mathrm{s}}$ on $b$ results only from changes in the bonds' mass with changes in their thickness, so this parameter is important for density only in the region of high values of $k$. Variations of the relative snow density over the possible range of $k$ and of $b$ are as follows: $\rho_{\mathrm{s}} / \rho_{\mathrm{i}}=0.07-0.22$ at $j=3 ; \rho_{\mathrm{s}} / \rho_{\mathrm{i}}=0.14-0.47$ at $j=4 ;$ and $\rho_{\mathrm{s}} / \rho_{\mathrm{i}}=0.21-0.72$ at $j=6$. Average values of $\rho_{\mathrm{s}} / \rho_{\mathrm{i}}$ at these coordination numbers are therefore $0.14,0.30$ and 0.50 , respectively. The latter value coincides precisely with relative density in case of cubical packing $j=6$ of spherical grains in contact with each other. At $\rho_{\mathrm{s}} / \rho_{\mathrm{i}} \leq 0.14$ the coordination number $j=3$ (or less). According to Figure 2, for 0.14 $\leq \rho_{\mathrm{s}} / \rho_{\mathrm{i}} \leq 0.22$ the coordination-number ice matrix can be 3 (if $k \leq 1.05$ ) or 4 (if $k \leq 1.25$ ). When the relative density is $0.22 \leq \rho_{\mathrm{s}} / \rho_{\mathrm{i}} \leq 0.47$, the coordination number may correspond to 4 (if $k \leq 1.15$ ) and to 6 (if $k \geq 1.05$ ).

In Equation (1) the contribution of the bonds to snow density is described by the expression in square brackets multiplied by $j / 2$. This component may reach $12 \%$ of the density at high coordination numbers $(j \geq 6)$ and high rigidity index $(b=0.5)$, and with loosely packed grains $(k=1.4)$, which are unlikely in granular snow. In real snow of medium density $b \leq 0.3$ and $k \leq 1.2$, where correction for bonds does not exceed $2-5 \%$, a simpler equation may be used to describe the snow density:

$$
\rho_{\mathrm{s}}=\rho_{\mathrm{i}} A \frac{\pi}{6} \frac{1}{k^{3}}
$$

It has previously been shown (Golubev, 1982) that snow mechanical properties, including Young's modulus $E$ and
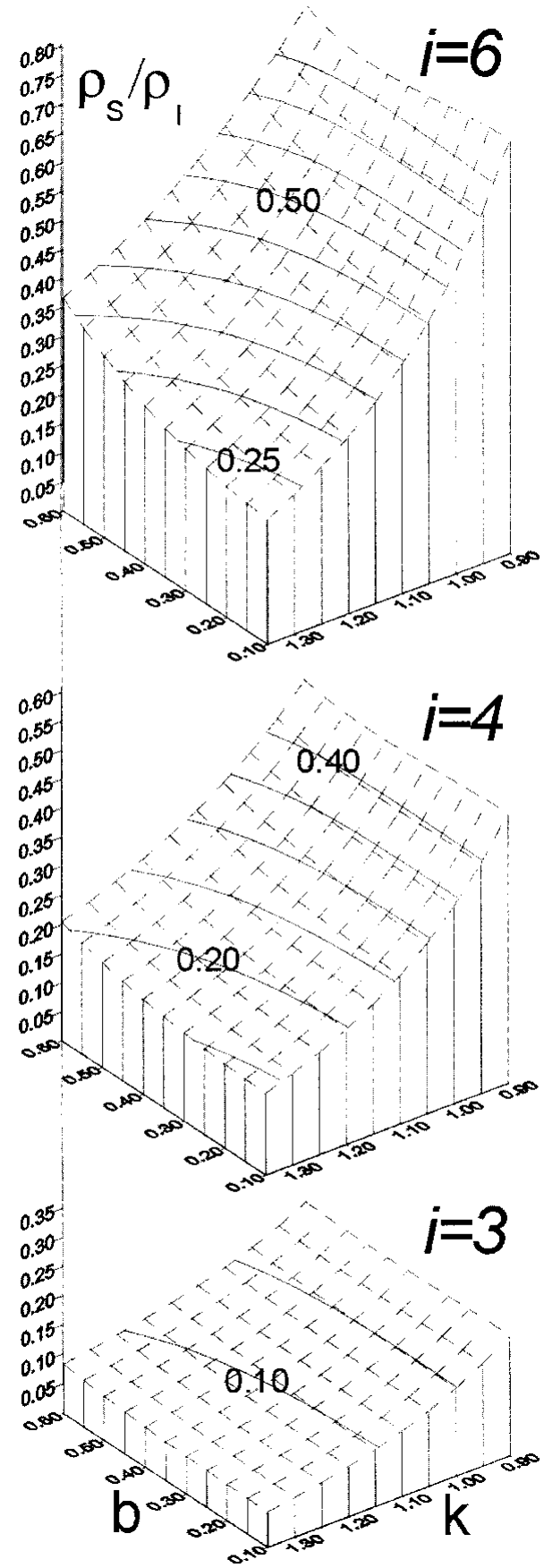

Fig. 2. Relative snow density vs structural parameters.

Poisson's ratio $\nu$, may be described by structural indexes as follows:

$$
\begin{aligned}
E_{\mathrm{s}}= & E_{\mathrm{i}} \frac{\rho_{\mathrm{s}}}{\rho_{\mathrm{i}}} \frac{1.5 k^{2} \cos \alpha}{\left(1+1.72 \sin ^{2} \alpha\right)}\left[\frac{\ln \left(1 / b^{2}\right)}{\sqrt{1-b^{2}}}+\frac{k-1}{b^{2}}\right]^{-1} \\
& \cdot\left\{1+\frac{j}{2}\left[1.5 k b^{2}+\left(1-b^{2}\right)^{3 / 2}-1\right]\right\}^{-1},
\end{aligned}
$$

and

$$
\nu_{\mathrm{s}}=\nu_{\mathrm{i}} k c\left[1+\frac{k-1}{b^{2}} \frac{\sqrt{1-b^{2}}}{\ln \left(1 / b^{2}\right)}\right]^{-1},
$$

where $E_{\mathrm{i}}$ and $\nu_{\mathrm{i}}$ are Young's modulus and Poisson's ratio of solid ice, $\alpha$ is standard deviation of the elements from the symmetry axes in regular packing $\left(\alpha=30^{\circ}\right.$ at $j=3, \alpha=$ $35^{\circ}$ at $j=4$ and $\alpha=0^{\circ}$ at $j \geq 6$ ) and $c$ is the parameter describing the lateral deformation of the medium as a function of the coordination number $j(c=(j-2) / 4$ at $j<6$ and 


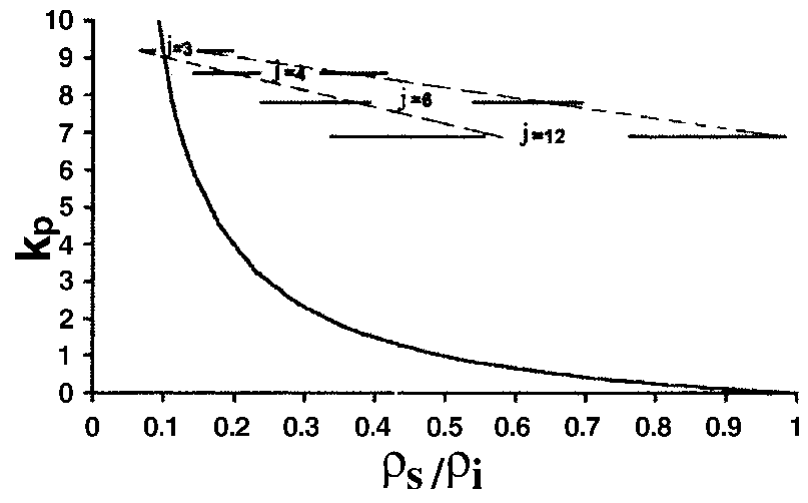

Fig. 3. Porosity factor $K_{\mathrm{p}}$ vs relative snow density. Solid horizontal lines show the ranges $\rho_{\mathrm{s}} / \rho_{\mathrm{i}}$ for various coordination numbers. Dashed lines show areas of $\rho_{\mathrm{s}} / \rho_{\mathrm{i}}$ at the most probable $k$ values (see text).

$c=1$ at $j \geq 6$ ). The expression in square brackets in Equation (3), as in Equation (1), describes the relationship between snow density and bonds' mass, and in most cases may be omitted as it differs insignificantly from $0(<5 \%)$.

It follows from Equations (1-4) and Figure 2 that changes in snow-texture indexes $k, b$ and $j$ must result in essential changes in density and in mechanical properties; the significance of any given parameter may vary with the snow density, which also implies changes in the pattern of the relationship between snow density and its properties, and therefore the stadial character of the gravitational compaction process.

\section{RESULTS AND DISGUSSION}

\section{The snow-compaction mechanism}

In granular snow a volume equal to $V_{\Sigma}=\left(\pi D^{3} / 6\right)\left(\rho_{\mathrm{i}} / \rho_{\mathrm{s}}\right)$ corresponds to each grain. The ice grain itself occupies only part of the volume $\left(V_{\mathrm{g}}=\pi D^{3} / 6\right)$, while share of pore space is $V_{\mathrm{p}}=\left(\pi D^{3} / 6\right)\left[\left(\rho_{\mathrm{i}} / \rho_{\mathrm{s}}\right)-1\right]$. The ratio between $V_{\mathrm{p}}$ and $V_{\mathrm{g}}$, and the ratio between pore volume in snow and that of the ice matrix itself, is the coefficient of porosity $K_{\mathrm{p}}=V_{\mathrm{p}} / V_{\mathrm{g}}=\left[\left(\rho_{\mathrm{i}} / \rho_{\mathrm{s}}\right)-1\right]$. According to Equation (2), this coefficient may be described as

$$
K_{\mathrm{p}}=\frac{6 k^{3}}{\pi A}-1 \text {. }
$$

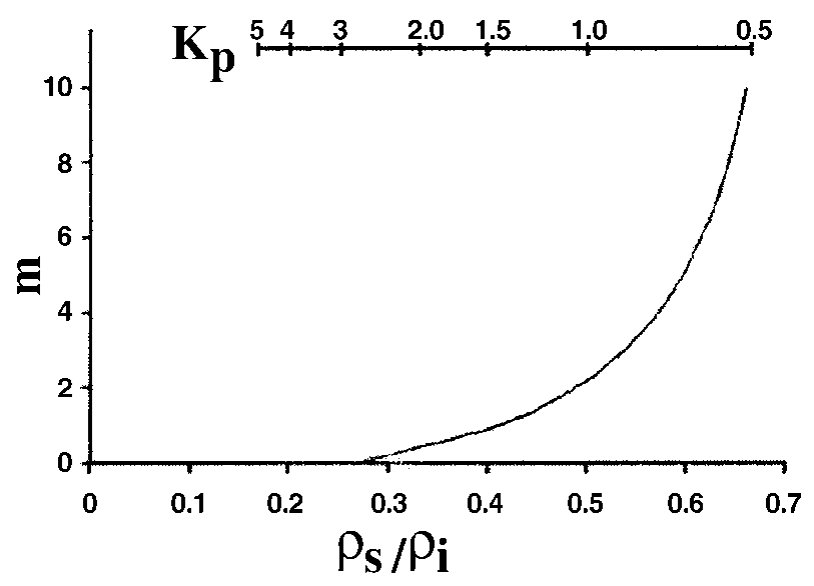

Fig. 4. $m$ (see text) vs $\rho_{\mathrm{s}} / \rho_{\mathrm{i}}\left(\right.$ or $\left.K_{\mathrm{p}}\right)$.
Figure 3 demonstrates the relationship between $K_{\mathrm{p}}$ and $\rho_{\mathrm{s}} / \rho_{\mathrm{i}}$ and gives the intervals of $\rho_{\mathrm{s}} / \rho_{\mathrm{i}}$ for some coordination number $j$ at $k=0.9-1.35$. At $j=3$ and $j=4$, with $k=0.95-$ 1.3 , the relative snow density may vary from 0.37 to 0.07 , while $K_{\mathrm{p}}$ lies within the range 3 to $>10$. Therefore, as the volume of pores surrounding each grain is several times greater than the volume of the grain itself, other grains from the overlying layer may easily displace (fall down) into the free space, resulting in rapid snow compaction. At $j=6$ and $k=0.95-1.15$, the relative density $\rho_{\mathrm{s}} / \rho_{\mathrm{i}}$ is $0.6-0.35$ and $K_{\mathrm{p}}=$ $0.5-2$. The pore volume around grains exceeds the volume of the grain only by a factor of 2 or less. Therefore, other grains from adjacent layers may migrate into pore space only if the packing of grains within a given layer changes its regularity

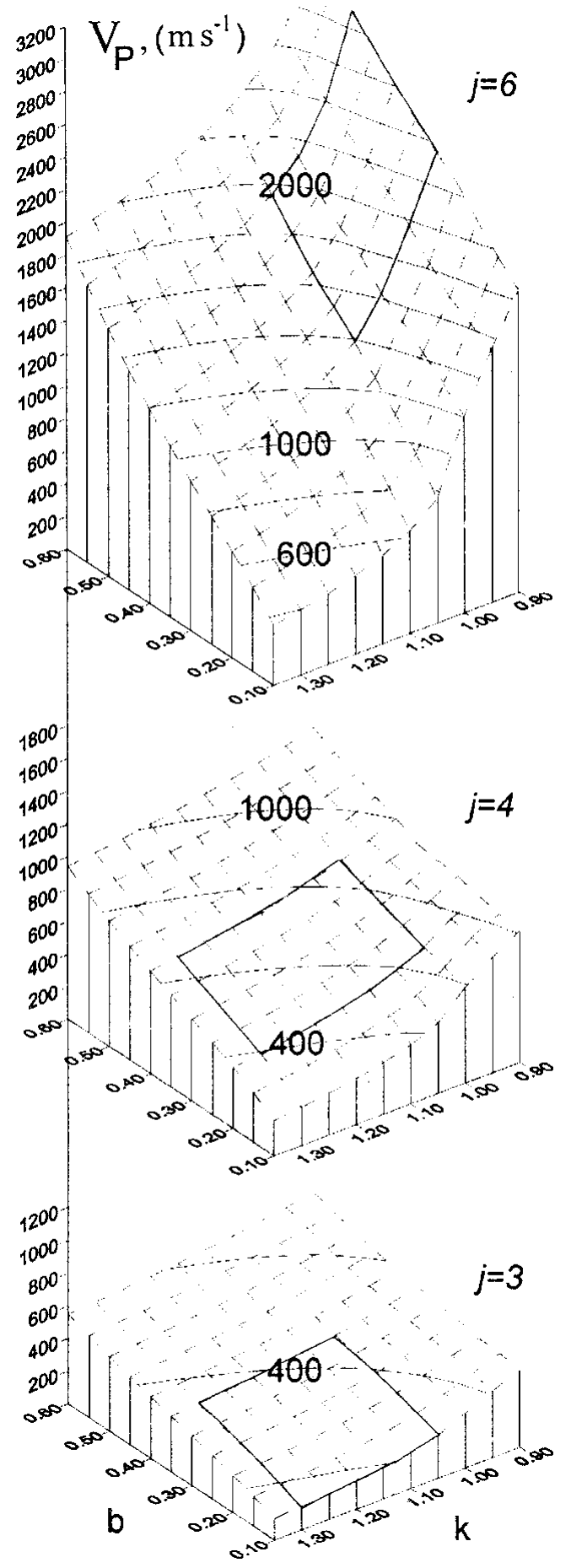

Fig. 5. Velocity of $P$-waves vs structural parameters. 


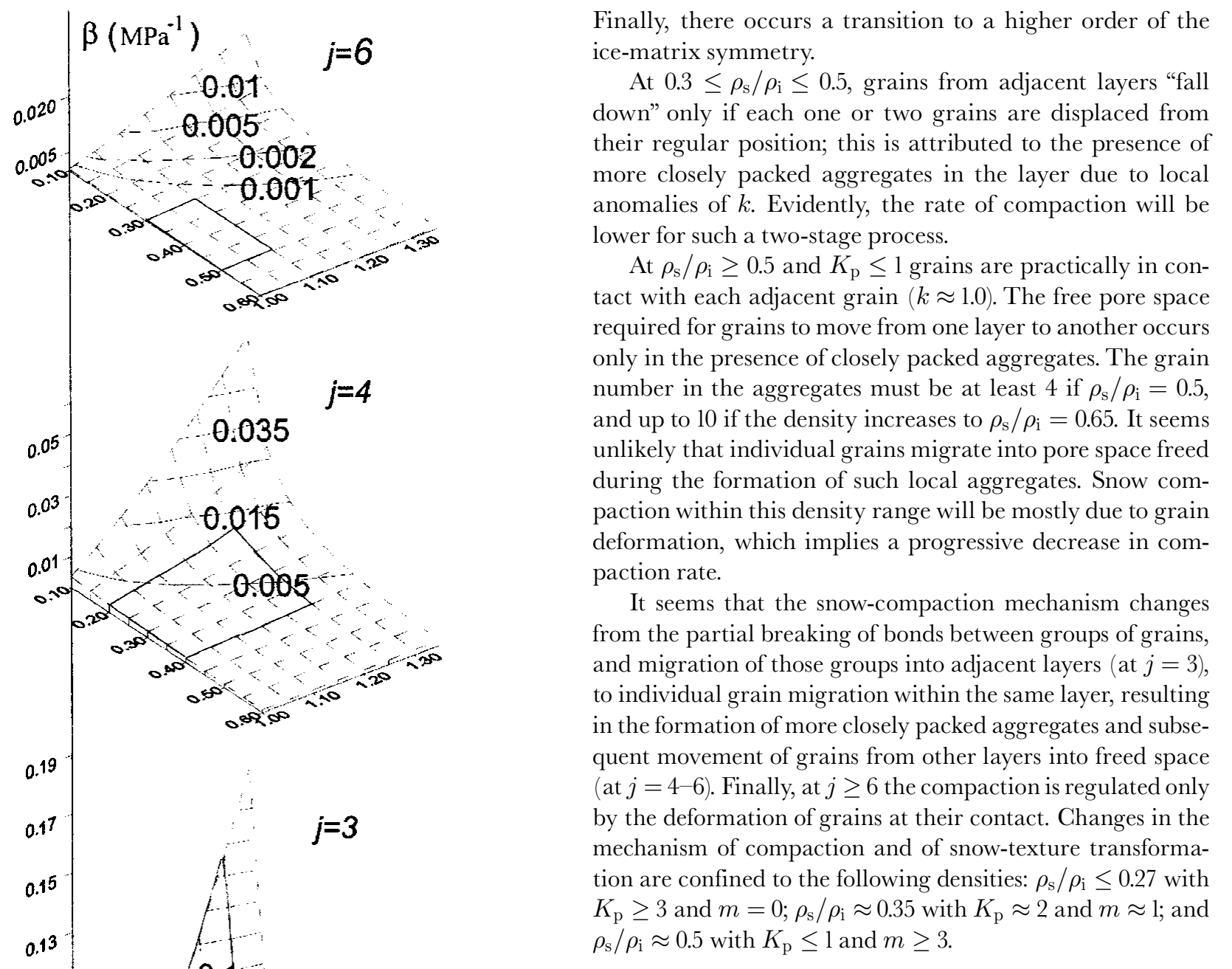

Changes in mechanical properties

Golubev (1982) and Golubev and Frolov (1998) applied the model to calculate Young's modulus, Poisson's ratio and the tensile strength of snow. The comparison between the calculated values and the experimental data showed sufficiently good agreement. A primary parameter, however, to be found by experiments is $\mathrm{P}$ - and $\mathrm{S}$-wave velocity in a medium. Therefore it seems worthwhile to analyze the model's potentialities calculating the wave velocities within snow using snow-structure parameters. As a first approximation, we take the familiar equation describing longitudinal wave velocities in a medium:

$$
V_{\mathrm{p}}=\sqrt{\frac{3(1-\nu)}{\rho_{\mathrm{m}} \beta(1+\nu)}}=\sqrt{\frac{E(1-\nu)}{\rho_{\mathrm{m}}(1+\nu)(1-2 \nu)}},
$$

where $E$ is Young's modulus, $\rho_{\mathrm{m}}$ is the density, $\beta$ is the volume compressibility coefficient and $\nu$ is Poisson's ratio. The relationships between Young's modulus and Poisson's ratio as a function of $j, k$ and $b$ (see Equations (3) and (4)) permit transformation of Equation (6) as follows:

$$
\begin{aligned}
V_{\mathrm{p}}= & {\left[\frac{E_{\mathrm{i}} \rho_{\mathrm{s}}}{\rho_{\mathrm{m}} \rho_{\mathrm{i}}} \frac{1.5 \cos \alpha}{\left(1+1.72 \sin ^{2} \alpha\right)} \frac{k^{2} \sqrt{1-b^{2}}}{Z \ln \left(1 / b^{2}\right)}\right.} \\
& \left.\cdot \frac{\left[1-\nu_{\mathrm{i}} k(j-2) / 4 Z\right]}{\left[1+\nu_{\mathrm{i}} k(j-2) / 4 Z\right]\left[1-2 \nu_{\mathrm{i}} k(j-2) / 4 Z\right]}\right]^{\frac{1}{2}},
\end{aligned}
$$

where

$$
Z=1+\frac{k-1}{b^{2}} \frac{\sqrt{1-b^{2}}}{\ln \left(1 / b^{2}\right)} .
$$

Results of calculations by this equation for different $k, b$ looseness $k$ decreases while coordination number $j$ grows. 
and $j$ (from loose to compact snow) are shown in Figure 5. The diagrams show the range of velocities $V_{\mathrm{p}}$ for snow with various $L$ and $d$ at fixed density. It is seen that, unlike the density, the elastic-wave velocity depends on both $k$ and $b$ in a similar way. The velocities at the most probable $k=1.2-0.95$ and $b=0.15-0.5$ (corresponding to natural snow) show good agreement with generalized data of seismic-acoustic studies of snow cover performed in Antarctica, Greenland and the Khibines on the Kola Peninsula, Russia (Frolov and Fedyukin, 1998). A comparison between velocity diagrams plotted for coordination numbers of grain packing equal to 3,4 and 6 reveals that they overlap to a certain extent. The reason is that during development of snow-medium texture in which a minor coordination number (e.g. 3 or 4) predominates, the more rigid elements of texture with a greater coordination number (e.g. 4 or 6 ) are also involved in the medium. This fits well with the concept of snow packing as a large open system and confirms the adequacy of the developed model of grain packing. An inverse problem may also be approached. Using the experimentally measured P- and $\mathrm{S}$-wave velocities and density, it is possible to estimate the snow-texture parameters and snow mechanical properties.

As in Equation (6), the velocity may be expressed in terms of the medium volume compressibility coefficient $\beta$. As snow is not a continuous medium but a differentially elastic one, it is essential to define its effective volume compressibility taking into account the solid constituents, the pores and the filling substance. Such an approach has been developed by M. Biot, F. Gassmann, W. Dobrynin and others (see, e.g., Bourbie and others, 1986; Dobrynin and others, 1991). In case of snow, which is a gas-saturated porous medium with practically no elastic bonds between solid and gaseous phases, volume compressibility coefficient $\beta$ is

$$
\beta \approx p \beta_{\mathrm{p}}+\beta_{\mathrm{i}}=\beta_{\mathrm{sk}},
$$

where $p$ is the porosity and $\beta_{\mathrm{p}}, \beta_{\mathrm{i}}$ and $\beta_{\mathrm{sk}}$ are the coefficients of volume compressibility for pores, ice and skeleton, respectively. Using Equations (3), (4) and (6), we can obtain an equation describing $\beta_{\mathrm{sk}}$ of snow in terms of its textural parameters:

$$
\beta=3 \frac{\left\{Z-\frac{\left[2 \nu_{\mathrm{i}} k(j-2)\right]}{4}\right\}\left\{1+\left[1.5 k b^{2}+\left(1-b^{2}\right)^{\frac{3}{2}}-1\right] \frac{i}{2}\right\}}{\frac{E_{\mathrm{i}} \rho_{\mathrm{s}}}{\rho_{\mathrm{i}}} \frac{1.5 \cos \alpha}{\left(1+1.72 \sin ^{2} \alpha\right)} \frac{k^{2} \sqrt{1-b^{2}}}{\ln \left(1 / b^{2}\right)}} .
$$

Results of calculations using Equation (9) are given in Figure 6 , which for the first time allows the prediction of changes in the volume compressibility of snow at different coordination numbers and varying $k$ and $b$ values. On the whole, this figure provides a reasonably accurate picture of the variations in snow rigidity. All the specific features mentioned with reference to $V_{\mathrm{p}}$ are typical of $\beta$, while calculated values of $\beta$ do not contradict the data obtained for porous ice $\left(\sim 10^{-4} \mathrm{MPa}^{-1}\right)$. From Figure 6 it can be seen that $\beta$ tends to $10^{-3} \mathrm{MPa}^{-1}$ in compact snow and increases to $10^{-1} \mathrm{MPa}^{-1}$ and more in loose granular snow. Figures 2, 5 and 6 show the areas with the most probable magnitudes of physical parameters $\left(\rho_{\mathrm{s}} / \rho_{\mathrm{i}}, V_{\mathrm{p}}\right.$ and $\left.\beta\right)$ based on analysis of $j, b$ and $k$ values (Golubev and Frolov,
1998). These areas are in good agreement with experimentally measured magnitudes for real snow.

\section{GONGLUSION}

The presented results of our modelling of dry granular snow texture and physical properties demonstrate the potential of the suggested model. The regular lattice of bounded grains adequately represents the changes in snow physical properties during compaction.

The snow-compaction mechanism includes both deformation of grains (which depends considerably on the bond rigidity $b$ and grain-packing index $j$ ) and grain displacement ("falling down" of individual grains and their groups) into free pore spaces in adjacent snow microlayers. During the process, more closely packed aggregates are formed and the texture parameters (first of all, $j$ and $k$ ) undergo rapid changes. The prevalence of a certain type of texture transformation is manifested in that the gravitation compaction of snow proceeds in several steps delimited by "critical" snow densities.

The suggested analytical relationships between snow density, elastic wave velocity, volume compressibility and snow-texture parameters cover the whole range of possible values. The most probable configuration of the ice matrix in the snow can be deduced both from the snow-texture investigations and from the experimental data on the physical properties, mainly on mechanical parameters.

\section{ACKNOWLEDGEMENTS}

We wish to thank P. B. Grebennikov and D. M. Frolov for important contributions to the preparation of the manuscript. The research was supported in part by the foundation "Russian Universities - Fundamental Research" and the Russian Fund for Basic Research (N98-05-64865).

\section{REFERENGES}

Bourbié, T., O. Coussy and B. Zinszner. 1986. Acoustique des milieux poreaux. Paris, Ed. Technip.

Dobrynin, V. M., B. Yu. Vandelshtein and D. A. Kozhevnikov. 1991. Petrofizika [Petrophysics]. Moscow, Nedra Press.

Frolov, A. D. 1998. Elektricheskiye i uprugiye svoystva merzlych porodi ildov [Electric and elastic properties of frozen earth materials]. Pushchino, Rossiyskoy Akademii Nauk. Izdatel'stvo Pushchinskogo Nauchnogo Centra.

Frolov, A. D. and I.V. Fedyukin. 1996. Akusticheskiye kharakteristiki snezhnoledyanych obrazonvanii plotnost'yu $30-917 \mathrm{~kg} \mathrm{~m}^{-3}$ [Acoustic characteristics of snow-ice formations in the density range from $\left.30-917 \mathrm{~kg} \mathrm{~m}^{-3}\right]$. In Yamshchikov, V. S., ed. Problemy geoakustiki: metodika i sredstva [Problems of geoacoustics: techniques and tools]. Moscow, Russian Acoustic Society, 181-184.

Frolov, A. D. and I.V. Fedyukin. 1998. Elastic properties of snow-ice formations in their whole density range. Ann. Glaciol., 26, 55-58.

Golubev, V. N. 1982. Zavisimost' uprugikh svoystv snega ot yego structury [Dependence of the elastic properties of the snow structure]. Mater. Glyatsiol. Issled. 44, 65-73.

Golubev, V. N. and A. D. Frolov. 1998. Modelling the change in structure and mechanical properties in dry-snow densification to ice. Ann. Glaciol., 26, $45-50$.

Maeno, N. 1978. The electrical behaviors of Antarctic ice drilled at Mizuho station, east Antarctica. Natl. Inst. Polar Res. Mem., Special Issue 10, 77-94.

Paterson, W. S. B. 1994. The physics of glaciers. Third edition. Oxford, etc., Elsevier. 